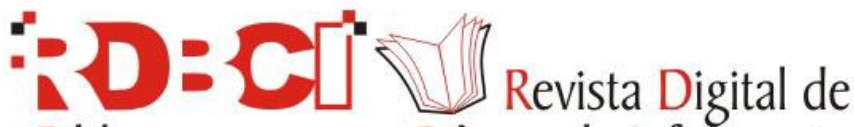 \\ Biblioteconomia e Ciência da Informação
}

ARTIGO

CDD: 001.42

\section{ABORDAGEM SENSE-MAKING NA CIÊNCIA DA INFORMAÇÃO: UMA BREVE CONTEXTUALIZAÇÃO}

SENSE-MAKING APPROACH IN THE INFORMATION SCIENCE: A BRIEF BACKGROUND

Marcio Gonçalves ${ }^{1}$

\section{RESUMO}

Este artigo busca esclarecer as definições da metodologia do Sense Making Approach, cunhado por Brenda Dervin, e evidenciar as possibilidades de seu uso na Ciência da Informação, a partir da citação de estudos nacionais que utilizaram esta metodologia como recurso para o alcance de seus objetivos.

\section{PALAVRAS-CHAVE}

Sense-Making. Brenda Dervin. Metodologia

\section{ABSTRACT}

This article seeks to clarify the definitions of the methodology of Making Sense Approach, coined by Brenda Dervin, and highlight the possibilities of its use in Information Science from the service of national studies that have used this methodology as a means to achieve their goals.

\section{KEYWORDS}

Sense-Making Approach. Brenda Dervin. Methodology

\footnotetext{
${ }^{1}$ Professor da Universidade Estácio de Sá, jornalista, mestre em Ciência da Informação e doutorando em Ciência da Informação pela UFRJ/IBICT. E-mail: marciog.goncalves@gmail.com.
}

(C) Rev. Dig. Bibl. Ci. Inf., Campinas, v.9, n.2, p.1-11, jan. /jun. 2012 - ISSN 1678-765X

http://www.sbu.unicamp.br/seer/ojs/index.php/sbu_rci/index 


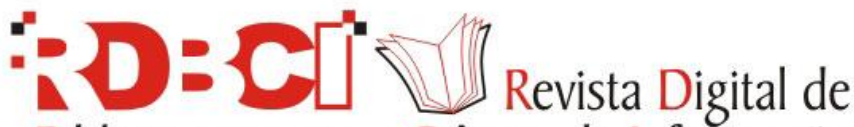 Biblioteconomia e Ciência da Informação}

ARTIGO

\section{A GÊNESIS DO SENSE-MAKING APPROACH}

Diante do pluralismo metodológico utilizado pela Ciência da Informação, a abordagem do Sense-Making tem se destacado como uma metodologia bastante presente nas pesquisas da área. De acordo com Bax e Dias (1997), a abordagem apresentada por Brenda Dervin $^{2}$, que é originária da área de Comunicação Social, "analisa os aspectos fundamentais inerentes às relações de interação entre seres humanos e sistemas”.

Segundo Ferreira (1997), dentre as atuais metodologias de estudos de usuários, esta tem se destacado como sendo a mais completa e abrangente, com grande número de adeptos em todo o mundo. O Sense-Making Approach, ou conforme tratado por Bax e Dias (1997) de "Construção de Sentidos", foi apresentado ao público pela professora do School of Communication da Ohio State University, Brenda Dervin, na International Communications Association Annual Meeting, em Dallas, Texas, nos Estados Unidos.

Ferreira (1997) lembra que esta metodologia se "propõe avaliar como pacientes/audiências/usuários/clientes/cidadãos percebem, compreendem, sentem suas interações com instituições, mídias, mensagens e situações" e usam a informação e outros recursos neste processo que tem como foco o fenômeno do sense-making.

Araújo, Pereira \& Fernandes (2009) destacam as seguintes temáticas que inicialmente foram abordadas por Dervin antes de a metodologia ganhar mais participação na Ciência da Informação: metodologia de pesquisa na comunicação, comunicação participativa e campanhas de comunicação pública. De acordo com os autores:

${ }^{2}$ Neste site é possível conhecer mais sobre os trabalhos de Brenda Dervin: < http://communication.sbs.ohiostate.edu/sense-making/default.html $>$.

(C Rev. Dig. Bibl. Ci. Inf., Campinas, v.9, n.2, p.1-11, jan. /jun. 2012 - ISSN 1678-765X

http://www.sbu.unicamp.br/seer/ojs/index.php/sbu_rci/index 
Aos poucos, a autora foi se aproximando da Ciência da Informação, principalmente a partir do desenvolvimento da chamada "metodologia Sense-Making", gradualmente construída pela autora desde 1972 e que ganhou uma formulação mais definitiva no início da década de 1980. Desde então, Dervin não cessou de problematizá-la e fazê-la avançar, por meio de textos teóricos e de muitas pesquisas de campo. (ARAÚJO, PEREIRA; FERNANDES, 2009)

Os mesmos autores relatam que essa "abordagem considera a informação como sendo uma construção do sujeito, a partir de suas experiências sociais, culturais, políticas e econômicas". Nesse sentido, a informação é subjetiva e só se torna significativa no contexto no qual está inserida.

\section{Para Ferreira (1997):}

A base conceitual do Sense-Making foi desenvolvida com suporte na teoria de vários estudiosos, como Bruner \& Piaget (cognição), Kuhn \& Habermas (constrangimento das ciências tradicionais e alternativas), Ascroft; Beltran \& Rolins (teórica crítica), Jackins \& Roger (teoria psicológica) e principalmente em Carter, teórico da comunicação, afirmando que o homem cria ideias para transpor as lacunas que lhes são apresentadas em decorrência da descontinuidade sempre presente na realidade. (FERREIRA, 1997)

O Sense-Making é um modo de tratar a necessidade de informação. De acordo com Rozados (2003), esta necessidade é focada na relevância "(relevância é o que produz sentido; relevante é o que tem sentido)". Para a autora, "no Sense-Making a busca de informação é orientada por um gap, uma falta, uma falha na estrutura de conhecimento do usuário. Por esta teoria, produzir sentido é lançar pontes para sanar esta falha.

Diante do exposto, Dervin (1992) conclui:

The term sense-making has come to be used to refer to a theoretic net, a set of assumptions and propositions, and a set of methods which have been developed to study the making of sense that people do in their everyday experiences. Some people call it a theory, others a set of methods, others a methodology, others a body of findings. In the most general sense, it is all of these.

(C) Rev. Dig. Bibl. Ci. Inf., Campinas, v.9, n.2, p.1-11, jan. /jun. 2012 - ISSN 1678-765X

http://www.sbu.unicamp.br/seer/ojs/index.php/sbu_rci/index 
Quando a autora se refere às raízes do Sense-Making ${ }^{3}$, ela afirma:

The term "Sense-Making" is a label for a coherent set of concepts and methods used in a now 8-year programmatic effort to study how people construct sense of their worlds and, in particular, how they construct information needs and uses for information in the process of sense-making. Since sense-making is central to all communicating situations, (whether they be intra-personal, interpersonal, mass, cross-cultural, societal, or international) the Sense-Making approach is seen as having wide applicability. (DERVIN, BRENDA, 1983)

\section{A CONTEXTUALIZAÇÃO DA ABORDAGEM}

Conforme abordado por Ferreira (10997), a abordagem do Sense-Making é bastante crescente nas áreas de comunicação, informação e biblioteconomia. Ela também tem sido usada na educação, na assistência social, na psicologia, na medicina e em outras. Quanto ao número da amostra de pesquisados, esse número vai desde 20 a cerca de 100 elementos. A autora cita que nas teses de doutoramento esta abordagem é mais presente e que suas aplicações estão nos contextos de pesquisa de opinião pública, de política, de processos de comunicação na área de saúde, de estudos acerca de imagens organizacionais, de recepção de audiência e, recentemente, de estudos sobre o uso de telecomunicações, além de uma variedade de níveis analíticos (individual, grupal, organizacional, comunitário, cultural).

Venâncio \& Nassif (2008) consideram que nos estudos de busca e uso da informação, a metodologia do Sense-Making é associada ao deslocamento da ênfase colocada nos sistemas de informação (correspondência entre uma busca de informação e a recuperação de documentos) para os usuários de informação (correspondência entre o sistema e a necessidade de informação). Esse direcionamento do enfoque para os usuários individuais é percebido em Dervin pela análise dos processos de busca e uso da informação como práticas comunicacionais produzidas e reproduzidas por diferentes indivíduos, nos diversos contextos em que atuam.

\footnotetext{
${ }^{3}$ De acordo com o apresentado por Ferreira (1997), conforme mensagem eletrônica, recebida por Dervin, desde final de 1994 o uso de letras maiúsculas é para se referir à abordagem e minúsculas para o fenômeno.

(C) Rev. Dig. Bibl. Ci. Inf., Campinas, v.9, n.2, p.1-11, jan. /jun. 2012 - ISSN 1678-765X

http://www.sbu.unicamp.br/seer/ojs/index.php/sbu_rci/index
} 
De acordo com os autores:

Um pressuposto básico da teoria reside na ideia de descontinuidade. Segundo Dervin (1992), a descontinuidade é um aspecto central da realidade, presente em todas as situações vivenciadas pelo ser humano. O indivíduo cria sentido para transpor os gaps cognitivos que lhes são apresentados em decorrência da descontinuidade sempre presente na realidade, incompleta e inconstante. (VENÂNCIO \& NASSIF, 2008, p. 97)

A figura abaixo contextualiza, conforme descrevem Bax \& Dias (1997), que a metodologia estuda quatro pontos fundamentais, presentes em todas as situações de busca de informação vividas por seres humanos. "Tais pontos podem ser melhores explicados através da metáfora de um indivíduo atravessando uma ponte". Os pontos são: a situação (S), as lacunas (L), os auxiliadores $(\mathrm{A})$ e as pontes $(\mathrm{P})$.

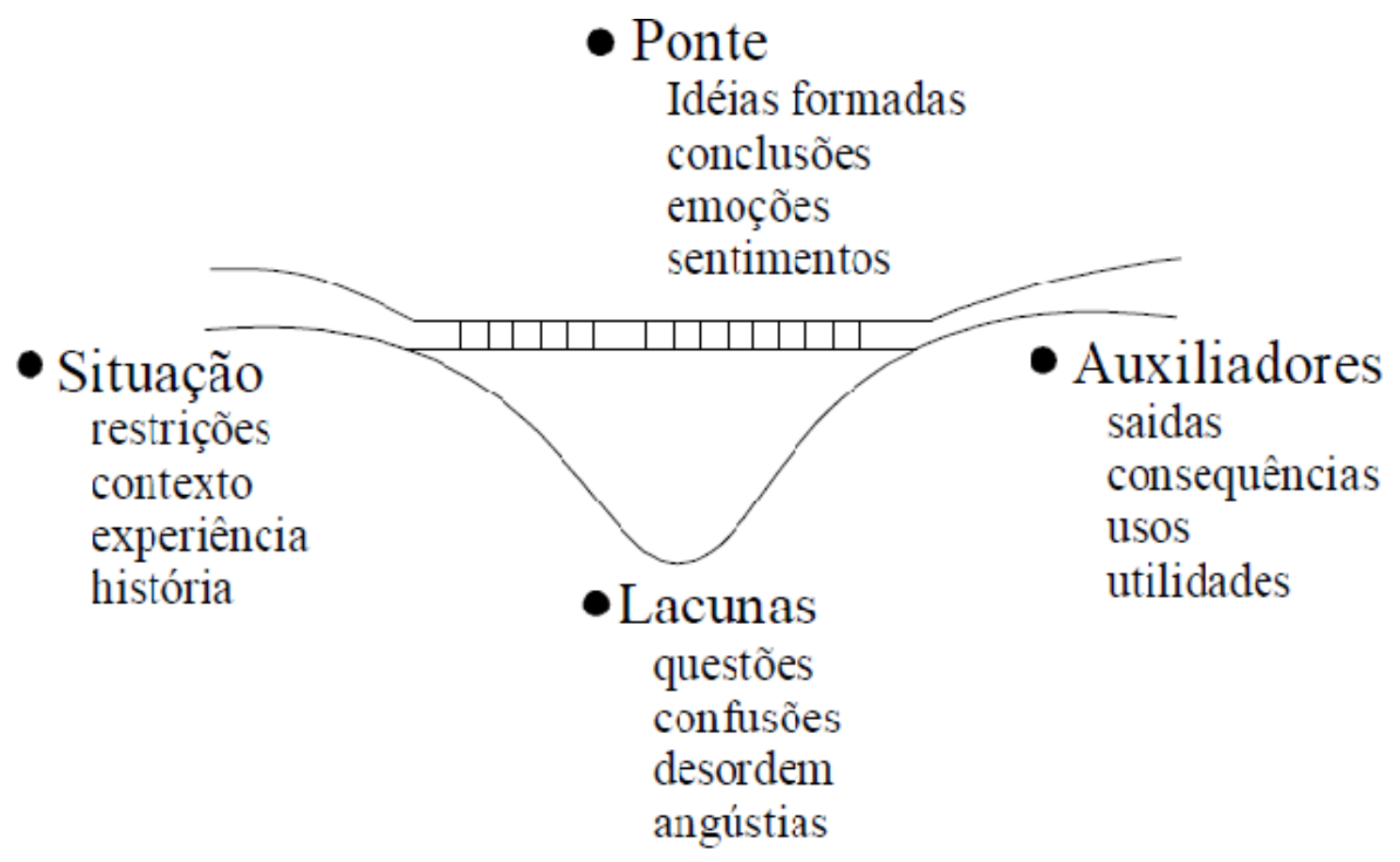

FIGURA 1 - Metáfora da Construção de Sentido FONTE - Bax; Dias, 1997 
Dada uma determinada situação de busca de informação, o ponto $\mathrm{S}$ é caracterizado por um conjunto de ideias e conceitos sob a influência das restrições, contexto, experiências e história do entrevistado.

Os "Ls" representam problemas ou lacunas do tipo questões, confusões, angústias, que impedem que a pessoa alcance os auxiliadores.

Os "As" são aqueles conceitos ou ideias que ajudarão a cruzar a ponte sobre as lacunas, ou seja, resultados, consequências, impactos, efeitos, usos e utilidade.

Os "Ps" são as ideias formadas, conclusões alcançadas, emoções e sentimentos que formam a ponte sobre as lacunas e ligam a situação aos auxiliadores.

No que tange ao estudo dos usuários, Miranda (2006) nos apresenta um quadroresumo com a citação de alguns outros autores que, além de Dervin, trabalham com a abordagem da construção de sentido. 


\begin{tabular}{|c|c|c|}
\hline ABORDAGEM & $\begin{array}{l}\text { AUTORES QUE } \\
\text { UTILIZARAM }\end{array}$ & CARACTERISTICAS DA ABORDAGEM \\
\hline \begin{tabular}{|l} 
Valor \\
Agregado
\end{tabular} & \begin{tabular}{l|} 
Taylor, \\
MacMullin, Hall, \\
Ford, Garvey, \\
Mohr, Paisley, \\
Farradane
\end{tabular} & $\begin{array}{l}\text { Foco na percepção da utilidade e valor que o usuário traz para o } \\
\text { sistema. Pretende fazer do problema do usuário o foco central, } \\
\text { identificando diferentes classes de problemas e ligando-os aos } \\
\text { diferentes traços que os usuários estão dispostos a valorizar } \\
\text { quando enfrentam problemas. É um trabalho de orientaçăo } \\
\text { cognitiva em processamento da informaçăo. (problema> valores } \\
\text { cognitivos }>\text { soluçóes) }\end{array}$ \\
\hline $\begin{array}{l}\text { Construção de } \\
\text { Sentido }\end{array}$ & \begin{tabular}{|l|} 
Dervin, Fraser, \\
Edelstein, \\
Grunig, Stamm, \\
Atwood, Palmor, \\
Palmour, Carter, \\
Dewdney, \\
Waner, Chen, \\
Burger, Hernon \\
\end{tabular} & $\begin{array}{l}\text { Conjunto de premissas conceituais e teóricas para analisar como } \\
\text { pessoas constroem sentido nos seus mundos e como elas usam a } \\
\text { informaçăo e outros recursos nesse processo. Procura lacunas } \\
\text { cognitivas e de sentido expressas em forma de questôes que } \\
\text { podem ser codificadas e generalizadas a partir de dados } \\
\text { diretamente úteis para a prática da comunicaçăo e informaçăo. } \\
\text { (situação > lacuna cognitiva e de sentido > uso) }\end{array}$ \\
\hline $\begin{array}{l}\text { Anomalia } \\
\text { Cognitiva }\end{array}$ & \begin{tabular}{|l|} 
Belkin, Oddy, \\
Ofori-Dwumfuo
\end{tabular} & $\begin{array}{l}\text { Foco nas pessoas em situações problemáticas, em visões da } \\
\text { situaçăo como incompletas ou limitas de alguma forma. Usuários } \\
\text { são vistos como tendo um estado de conhecimento anômalo, no } \\
\text { qual é difícil falar ou mesmo reconhecer o que está errado, e } \\
\text { enfrentam lacunas, faltas, incertezas e incoerências, sendo } \\
\text { incapazes de especificar o que é necessário para resolver a } \\
\text { anomalia. } \\
\text { (situação anômala > lacunas cognitiva > estratégias de busca) }\end{array}$ \\
\hline
\end{tabular}

Fonte: MIRANDA (2006, p. 100)

Podemos perceber que nesta primeira década do século XXI, os estudos de usuários estão voltados tanto para o comportamento informacional, quanto para a avaliação de satisfação e desempenho, enfatizando a relação entre usuários e sistemas de informação interativos, no contexto social das tecnologias de informação e comunicação.

Neste sentido, Venâncio \& Nassif (2008), dizem que a teoria enfatiza:

(a) As situações experienciadas pelos indivíduos em um contexto temporal e espacial no qual surgem as necessidades de informação influenciadas pela experiência e pelas histórias de vida do indivíduo;

(b) Os gaps cognitivos enfrentados (necessidades de informação, questões que as pessoas têm quando constroem sentido e movem-se através do tempo-espaço) que são representados pelas angústias, desordens e confusões; 
(c) O uso da informação, ou seja, as pontes ou estratégias construídas (idéias, pensamentos, atitudes) para superação dos gaps.

\section{O SENSE-MAKING APPROACH NA PRÁTICA DE CIÊNCIA DA INFORMAÇÃO NO BRASIL: CONSIDERAÇÕES}

Na Ciência da Informação, Araújo, Pereira \& Fernandes (2009, buscaram referências à Brenda Dervin em todos os artigos publicados em sete periódicos nacionais, entre os anos de 2003 e 2007. Os autores constataram que Dervin é citada em 27 dos 872 artigos analisados. Das 19577 referências bibliográficas destes artigos, 42 são de trabalhos da pesquisadora. A obra mais citada, Information needs and uses, teve 16 referências. A maior contribuição da autora se dá no âmbito dos estudos de usuários da informação e a instituição brasileira que mais a cita é a Universidade Federal de Minas Gerais.

Um destes exemplos é o trabalho de Venâncio \& Nassif (2008), em que investigaram o comportamento de busca de informação de pessoas responsáveis pela tomada de decisão organizacional aplicando a metodologia de Dervin.

Lira, Cândido, Araújo \& Barros (2007) utilizaram o método para avaliar, no âmbito organizacional, os principais desafios para as pessoas e paras as organizações em saber detectar e gerenciar a informação de forma eficaz. Alves e Faqueti (2002) analisaram as mudanças no Serviço de Referência sob o impacto das novas tecnologias de informação. A revisão da literatura centrada no usuário aponta para uma maior intervenção dos bibliotecários no processo de busca de informação.

Costa e Ramalho (2010) também se valeram do Sense-Making para descrever os sistemas interativos de informação pela interface com os usuários da informação, sob a perspectiva da interação homem-computador. Na mesma linha, o estudo de Pereira (2002) analisou alguns aspectos da interação ser humano-computador e desta metodologia para examinar um site de uma empresa no que tange ao leiaute das sucessivas telas com janelas, menus, botões, ícones e todos os componentes do sistema visíveis para o usuário. 
Ferreira e Reis (2008) analisaram o perfil do profissional que trabalha com a arquitetura de informação no Brasil (pesquisa quantitativa por meio de um questionário on-line) e as dificuldades, técnicas e metodologias encontradas nos seus projetos (pesquisa qualitativa por meio de entrevistas em profundidade com apoio da abordagem do Sense-Making). Nascimento (2011), ao descrever a origem, histórico e desenvolvimento dos estudos de usuários, particularmente no Brasil na década de 1970, identifica o Sense-Making como uma das metodologias utilizadas.

\section{CONSIDERAÇÕES FINAIS}

A intenção do Sense-Making é fornecer orientações gerais para assegurar que o diálogo seja incentivado em todos os aspectos da comunicação. É uma metodologia para a prática comunicacional. Dar voz ao usuário, quando se quer entender as necessidades e usos que estes indivíduos fazem com a informação, permite a criação de uma comunicação dialógica.

A base teórica para a construção desta metodologia de pesquisa, por ter buscado referência na ciência da cognição, na teoria crítica, na terapia psicológica e, principalmente, na teoria da comunicação, demonstra que a informação não é algo que exista independente e externamente ao ser humano. Pelo contrário, a informação é um produto da observação humana.

As redes sociais na internet e as mídias sociais têm permitido maior participação das pessoas na construção de informação. Como a metodologia é um modo de tratar a necessidade de informação, com base na relevância, ou seja, na informação que produz sentido, o Sense-Making ganha espaço, por exemplo, para ser aplicado em pesquisas que analisam a informação e o comportamento do usuário no uso de plataformas sociais digitais.

Em uma sociedade que está cada vez mais interconectada, os sistemas de informação e as mídias digitais passam a incluir o usuário nas suas criações. Usado em entrevistas qualitativas ou quantitativas, o Sense-Making permite que instituições possam usar esta metodologia para aprender o que não sabiam e poder mudar suas expectativas. As pessoas, neste 




ARTIGO

caso, ficam em primeiro plano. Ouvi-las, portanto, contribui para a formação de sistemas de informação interativos mais eficazes.

\section{REFERENCIAS}

ALVES, M. B. M.; FAQUETI, M. F. Mudanças no serviço de referência, em bibliotecas universitárias, sob o impacto das novas tecnologias. Disponível em <http://www.sibi.ufrj.br/snbu/snbu2002/oralpdf/89.a.pdf>. Acesso em: 16 julho 2010.

ARAÚJO, C. A; PEREIRA, G. A.; FERNANDES, J. R. A contribuição de B. Dervin para a Ciência da Informação no Brasil. Bibli: R. Eletr. Bibliotecon. Ci. Inf., Florianópolis, Santa Catarina v. 14, n. 28, p.57-72, 2009.

BAX, M. P.; DIAS, E. W. A abordagem “Construção de Sentido". Relatório. Disponível em <http://www.bax.com.br/Bax/CVs/Disciplinas/UsuInfo/sensema6.html>. Acesso: 16 julho 2010.

COSTA, L. F.; RAMALHO, F. A. A usabilidade nos estudos de informação: em cena usuários e sistemas interativos de informação. Perspectivas em Ciência da Informação, v. 15, n.1, p. 93117, jan./abr, 2010.

DERVIN, B. From the mind's eye of the user: the sense-making qualitative-quantitative methodology. In: Glazier, Jack D; Powell, Ronald R. Qualitative Research in Information Management. Englewood, CO: Libraries Unlimited, pp. 61-84, 1992.

. An overview of sense-making research: concepts, methods, and results to date. International Communication Association annual meeting, Dallas, Maio, 1983. Disponível em <https://www.ideals.illinois.edu/bitstream/handle/2142/2281/Dervin83a.htm> Acesso 25 nov. 2011.

FERREIRA, S. M. S. P; REIS, G. A prática de Arquitetura de Informação de websites no Brasil. TransInformação, Campinas, v. 20, n. p. 285-307, set./dez., 2008.

LIRA, W.S.; CANDIDO, G. A; ARAUJO, G. M. B. Processo de decisão do uso da informação. Perspectivas em Ciência da Informação, v. 12, n.2, pp. 64-80, 2007.

MIRANDA, S. Como as necessidades de informação podem se relacionar com as competências informacionais. Ciência da Informação, Brasília, v. 35, n. 3, p. 99-114, 2006.

(C) Rev. Dig. Bibl. Ci. Inf., Campinas, v.9, n.2, p.1-11, jan. /jun. 2012 - ISSN 1678-765X

http://www.sbu.unicamp.br/seer/ojs/index.php/sbu_rci/index 
NASCIMENTO, M. J. Usuário da informação como produção científica e disciplina curricular: origem dos estudos e o ensino no Brasil. Revista Digital de Biblioteconomia e Ciência da Informação, Campinas, v.8, n. 2, p. 41-71, jan./jun. 201.

PEREIRA. E. C. Design de sistemas de informação centrado no usuário e a abordagem SenseMaking. TransInformação, Campinas, v. 14, n. 2 p. 139-151, jul./dez., 2002.

ROZADOS, H. B. F. A Ciência da Informação em sua aproximação com as Ciências Cognitivas. Em Questão, Porto Alegre, v.9, n.1, p. 79-94, jan./jun, 2003.

VENÂNCIO, L.; NASSIF, M. E. O comportamento de busca de informação sob o enfoque da cognição situada: um estudo empírico qualitativo. Ciência da Informação, Brasília, v. 37, n. 1, p. 95-106, jan./abr, 2008. 